\title{
Join the London Mathematical Society (LMS)
}

\section{Elizabeth Fisher (London Mathematical Society, London, UK)}

\section{About the LMS}

As a UK-wide learned society for mathematics, the London Mathematical Society (LMS) advances, disseminates and promotes mathematical knowledge, both nationally and internationally. Its activities include:

- Grants to support conferences and collaborative research between UK based and non-UK based mathematicians, e.g. Research in Pairs (https://www.lms. ac.uk/grants/research-pairs-scheme-4).

- Training opportunities aimed at Young Mathematicians and Early Career Researchers, e.g. LMS-CMI Research Schools: https://www.lms.ac.uk/events/lmscmi-research-schools.

- Prestigious prizes to recognise achievements in mathematics research, with nine Whitehead Prize winners going on to win EMS Prizes: https://www.lms.ac.uk/ prizes.

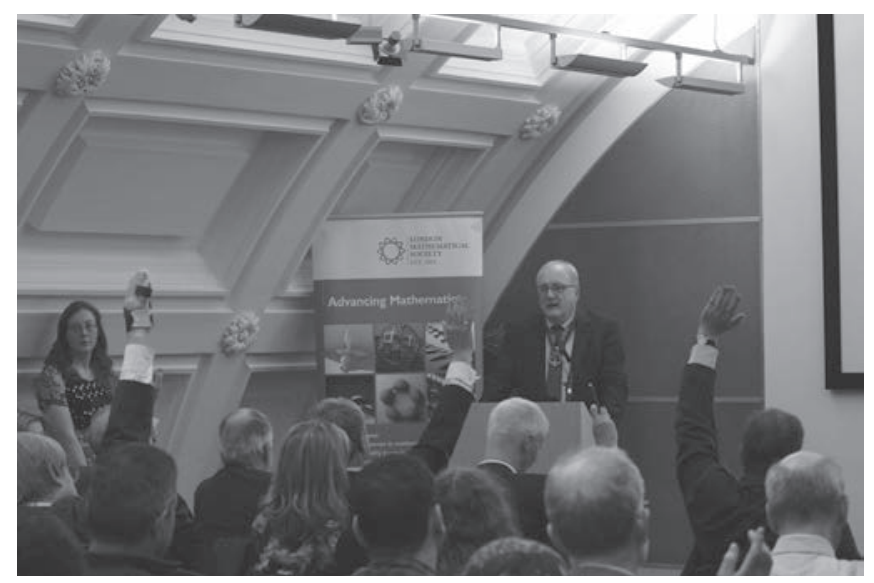

LMS Members at the Annual General Meeting, London, November 2016.

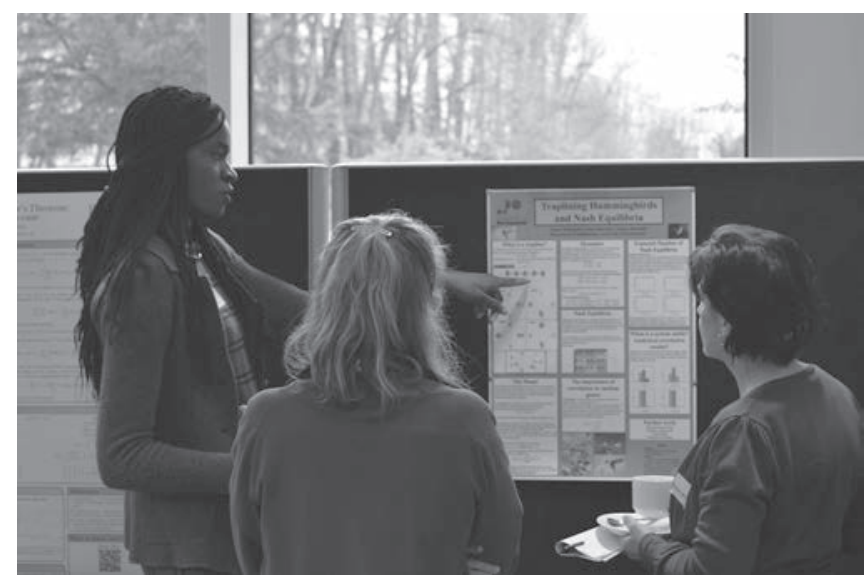

Participants at the LMS Women in Mathematics Day, Edinburgh, April 2016.
- Thirteen international peer-reviewed journals, seven of which are in collaboration with other learned societies and institutions, and two book series (lecture notes and student texts): https:/www.lms.ac.uk/publications.

- Scientific lectures and meetings for research mathematicians, including meetings at the ECM and the ICM: https://www.lms.ac.uk/events/society-meetings.

- Representation of mathematics research and education to Government and other national policymakers and sponsors: https://www.lms.ac.uk/policy/policy-consultations.

- Participation in international mathematical initiatives and promoting the discipline more widely, e.g. supporting mathematics in Africa through Mentoring African Research in Mathematics (MARM) and grants to conferences at the African Mathematics Millennium Science Initiative (AMMSI): https://www.lms.ac.uk/ grants/international-grants\#Africa.

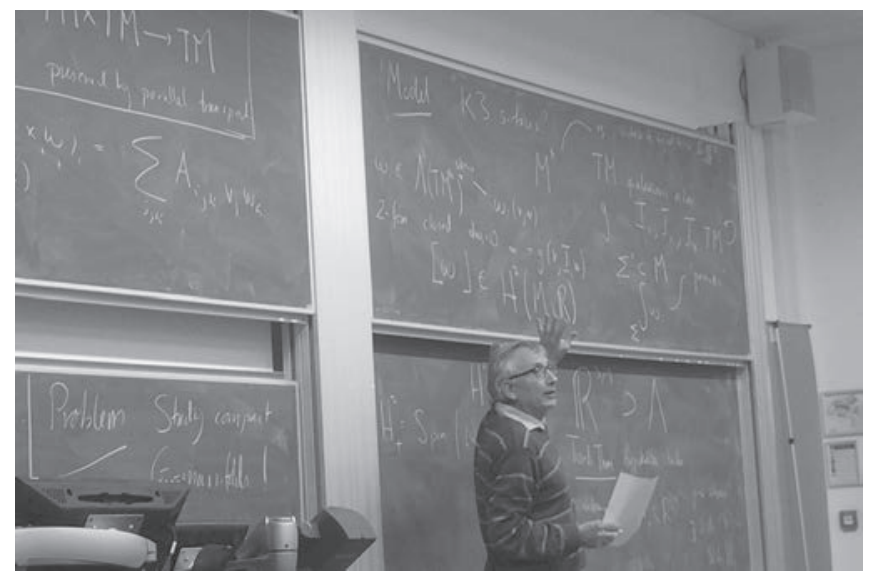

Simon Donaldson gives a talk at the South West \& South Wales Regional Meeting, Bath, December 2016.

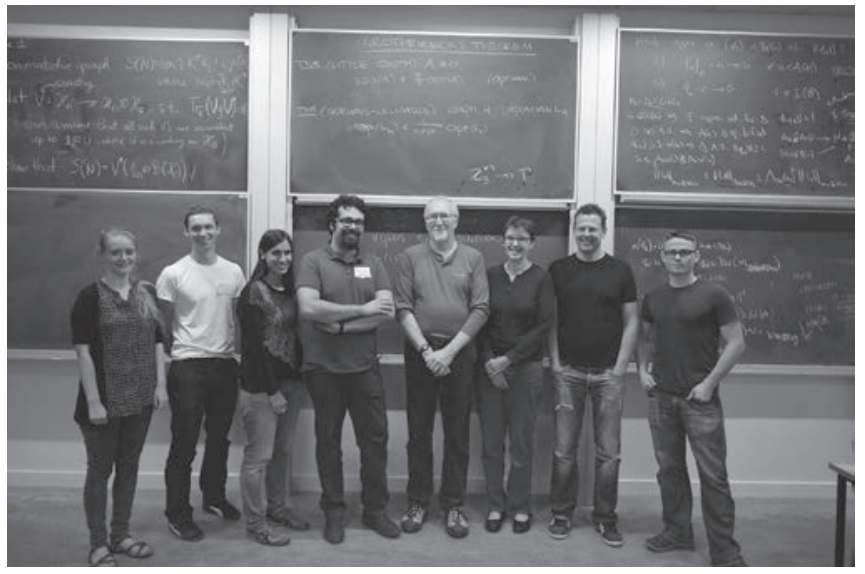

Speakers at the Research School, Belfast, September 2016. 
Further information about recent LMS activities can also be found in the Society's 2015-16 Annual Review: www. lms.ac.uk/sites/lms.ac.uk/files/files/files/reports/LMS \%20 Annual\%20review\%202016\%20web.pdf.

\section{The LMS and the European Mathematical Society}

The LMS has been a member society of the EMS since 1990. The collaboration between the LMS and the EMS has included:

- Hosting the EMS Ethics Committee in 2013 and both the EMS Executive Committee and the EMS Applied Mathematics Committee in 2014.

- Travel Grants to European Congresses of Mathematics.

- Participation of LMS delegates at the EMS Council Meetings.

This partnership was recently celebrated at a Joint Meeting held at the University of Birmingham in September 2015 to mark the societies' respective anniversaries: 25 years for the EMS and 150 years for the LMS.

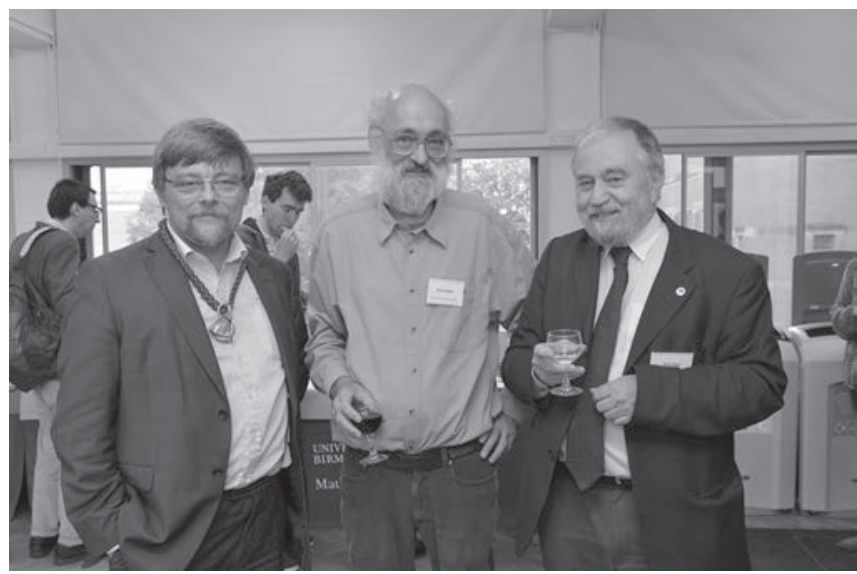

LMS Immediate Past President Terry Lyons FRS (Oxford), EMSLMS Meeting Organiser Chris Parker (Birmingham) and EMS President Pavel Exner (Academy of Sciences of the Czech Republic) at the Joint LMS-EMS Anniversary Meeting, Birmingham, September 2015.

\section{Membership of the LMS}

The LMS has a membership of 2,800 members that make up a vibrant international mathematical community, with $20 \%$ of the LMS membership based outside the UK, including 234 members based in other European countries.

Membership of the LMS falls into three categories:

- Ordinary membership: for academic staff, mathematicians in other occupations and all those with a deep commitment to mathematics and an interest in mathematical research.

- Associate membership: for undergraduates, postgraduates and early career mathematicians who are within three years of completing their PhD.

- Reciprocity membership: for those not normally resident in the UK and also members of some overseas mathematical societies.
The LMS is pleased to have reciprocal agreements with 20 mathematical societies. Any non-UK based EMS members thinking of joining the LMS are advised to check the list to see if their society is included when applying: https://www.lms.ac.uk/membership/member ship-categories\#Reciprocity.

\section{How to join}

The LMS welcomes applications via its online form: https://www.lms.ac.uk/membership/online-application, and applicants are formally elected to membership at one of the society meetings of the LMS.

Do visit the website for full details on the application process (https://www.lms.ac.uk/membership/howjoin) and the current fees (https://www.lms.ac.uk/sites/ lms.ac.uk/files/Membership/Subscription \%20Rates \%20 and \%20Notes \%202016-17\%20updated.pdf).

\section{Benefits of LMS membership include:}

- Membership of a vibrant, national and international mathematics community.

- Networking opportunities.

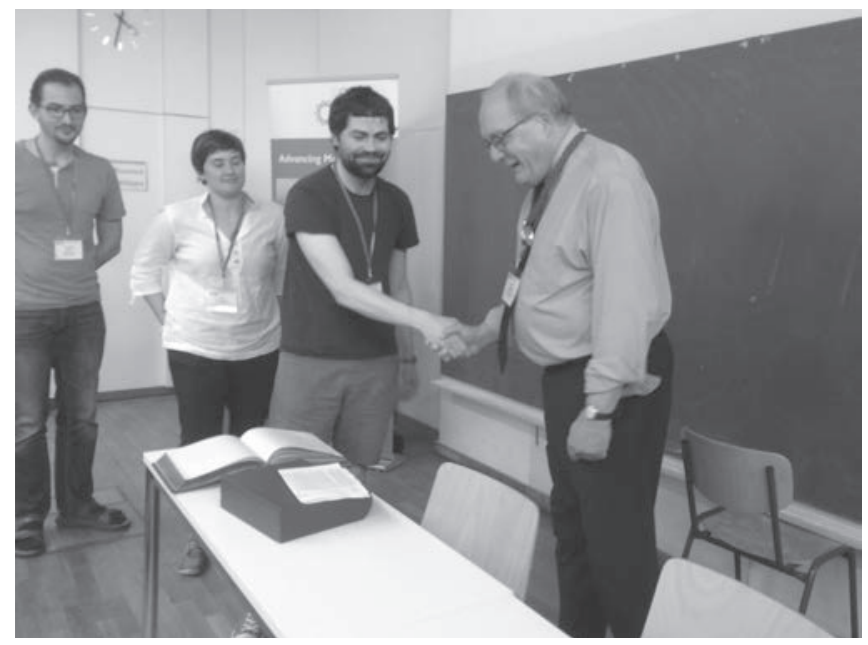

LMS President Simon Tavaré (Cambridge) welcomes new LMS members at the 7ECM in Berlin, July 2016

- Opportunities to influence national policy.

- Full voting rights in society elections - your chance to shape the future of the LMS.

- A complimentary monthly newsletter - available in print and online.

- Regular members-only LMS e-Updates.

- Opportunities to attend events hosted by the society.

- Free online subscriptions to the Bulletin of the London Mathematical Society, the Journal of the London Mathematical Society, the Proceedings of the London Mathematical Society and Nonlinearity (published jointly with the Institute of Physics).

- Members discount on other selected LMS publications: $25 \%$ discount on the LMS Lecture Note Series and $25 \%$ discount on LMS Student Texts.

- Use of the Verblunsky Members' Room at De Morgan House, Russell Square, London. 
- Use of University College London Library, where the society's library is housed.

- The opportunity to sign the LMS Members' Book, which dates back to 1865 when the society was founded and which contains signatures of members throughout the years, including Augustus De Morgan, Henri Poincaré, G. H. Hardy and Mary Cartwright.

\section{Contact the LMS}

We would be pleased to hear from you. For queries about membership, do email us at membership@lms.ac.uk and for regular news from the LMS, follow us on Twitter @ LondMathSoc.

\section{And what about Brexit?}

Following the Brexit vote, the LMS would like to express its support and solidarity with the $31 \%$ of the UK mathematical academic community from other EU states. We would like to reassure our EU friends and colleagues, wherever they are based, that we are keen to maintain our close mathematical and professional relationships with the rest of Europe, however the Brexit process proceeds. The EU referendum statement from the Council for the Mathematical Sciences (CMS), of which the LMS

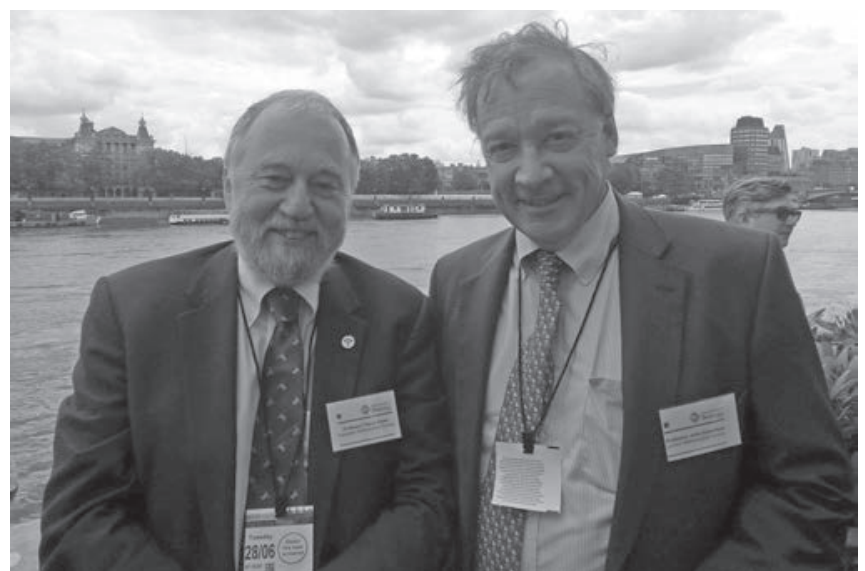

EMS President Pavel Exner and LMS Vice-President John Greenlees at the UK Parliamentary Links Day with Science, June 2016.

is a member, is also available at https://www.lms.ac.uk/ news-entry/05072016-1201/council-mathematical-sciences-eu-referendum-statement.

Elizabeth Fisher LMS Membership \& Activities Officer 